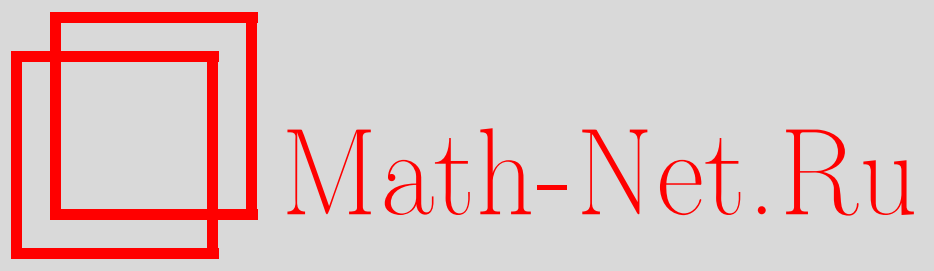

Дж. Губбиотти, Д. Леви, К. Шимитерна, Линеаризуемость и ложные пары Лакса для нелинейных неавтономных квад-графовых уравнений, подчиняющихся условию согласованности при обходах вокруг кубической ячейки, ТМФ, 2016, том 189, номер 1, 69-83

DOI: https://doi.org/10.4213/tmf9079

Использование Общероссийского математического портала Math-Net.Ru подразумевает, что вы прочитали и согласны с пользовательским соглашением http: //www . mathnet.ru/rus/agreement

Параметры загрузки:

IP: 18.234 .197 .8

26 апреля 2023 г., $07: 34: 30$

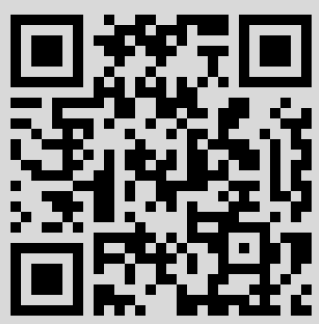




\title{
ЛИНЕАРИЗУЕМОСТЬ И ЛОЖНЫЕ ПАРЫ ЛАКСА ДЛЯ НЕЛИНЕЙНЫХ НЕАВТОНОМНЫХ КВАД-ГРАФОВЫХ УРАВНЕНИЙ, ПОДЧИНЯЮЩИХСЯ УСЛОВИЮ СОГЛАСОВАННОСТИ ПРИ ОБХОДАХ ВОКРУГ КУБИЧЕСКОЙ ЯЧЕЙКИ
}

\begin{abstract}
Обсуждается линеаризация неавтономных нелинейных уравнений в частных разностях, попадающих в классификацию Болла уравнений на квад-графах, подчиняющихся условиям согласованности при обходах вокруг элементарного куба решетки. Показано, что пара Лакса таких уравнений оказывается ложной. Приводятся обобщенные симметрии этих уравнений, которые оказываются неавтономными и зависящими от произвольной функции зависимых переменных, заданных в двух точках решетки. Эти обобщенные симметрии задаются дифференциально-разностными уравнениями, допускающими в некоторых случаях специфические преобразования Беклунда.
\end{abstract}

Ключевые слова: уравнения в частных разностях, $C$-интегрируемость, преобразование Беклунда, ложные пары Лакса.

DOI: $10.4213 /$ tmf9079

\section{1. ВВЕДЕНИЕ}

Симметрии и коммутирующие потоки лежат в основе теории интегрируемости. Преобразования Беклунда и нелинейные законы суперпозиции [1], [2] проложили дорогу к построению дискретизаций интегрируемых систем.

Работа Д. Леви и К. Шимитерна была частично поддержана грантом Italian Ministry of Education and Research, 2010 PRIN "Непрерывная и дискретная нелинейная интегрируемая эволюция: от волн на поверхности воды до симплектических отображений”. Работа Дж. Губбиотти и Д. Леви была поддержана грантом INFN IS-CSN4 "Математические методы нелинейной физики".

*Dipartimento di Matematica e Fisica, Università degli Studi Roma Tre, Roma, Italy

${ }^{\dagger}$ Instituto Nazionale di Fisica Nucleare, Sezione di Roma Tre, Roma, Italy.

E-mail: gubbiotti@mat.uniroma3.it,scimiterna@fis.uniroma3.it,decio.levi@roma3.infn.it 


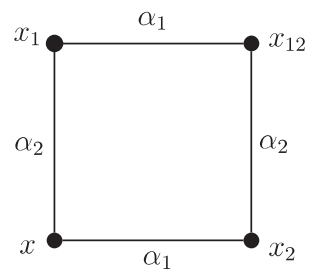

Рис. 1. Квад-граф.

Первая версия критерия интегрируемости, получившего название согласованности при обходе вокруг элементарного куба (критерий САС), содержится в работе Доливы и Сантини [3].

В последнее время с помощью критерия САС были получены многочисленные результаты, касающиеся классификации нелинейных разностных уравнений. Важность этого критерия обусловлена тем, что он обеспечивает существование преобразований Беклунда [4]-[7] и, следовательно, существование представления нулевой кривизны, или пары Лакса, которые, как хорошо известно [8], связаны как с линеаризуемыми, так и с интегрируемыми уравнениями.

Первая попытка построения классификации уравнений в частных разностях с помощью критерия САС была предпринята в работе [9] в предположении о том, что уравнения на всех гранях куба имеют один и тот же вид. В результате был получен класс дискретных уравнений, образуемых системами на квад-графах; базисными строительными блоками этих систем оказываются уравнения на четырехугольниках вида

$$
A\left(x, x_{1}, x_{2}, x_{12} ; \alpha_{1}, \alpha_{2}\right)=0,
$$

в которых четыре поля $x, x_{1}, x_{2}$ и $x_{12} \in \mathbb{C}$ соответствуют четырем вершинам четырехугольника (ниже в уравнении (3) эти поля обозначаются как $x_{1}, x_{2}, x_{4}$ и $x_{4}$, а на решетке с индексами $m$ и $n$ - как $x_{m, n}, x_{m+1, n}, x_{m, n+1}$ и $\left.x_{m+1, n+1}\right)$, а параметры $\alpha_{i} \in \mathbb{C}, i=1,2$, соответствуют ребрам четырехугольника $\left(\alpha_{2}=\alpha_{2}(n), \alpha_{1}=\alpha_{1}(m)\right)$ (см. рис. 1). В приведенных выше обозначениях, если четырехугольник получается с помощью выбора двух независимых направлений 1,2 , исходящих из начальной вершины, которой ставится в соответствие поле $x$, то нижние индексы 1 и 2 обозначают поля в вершинах со сдвигами на $\alpha_{1}$ и $\alpha_{2}$ вдоль направлений 1 и 2 , отсчитывая от начальной вершины, а нижний индекс 12 относится к полю, соответствующему последней, четвертой вершине четырехугольника. Кроме того, предполагается, что функция $A\left(x, x_{1}, x_{2}, x_{12} ; \alpha_{1}, \alpha_{2}\right)$ аффинно линейна по каждому из своих аргументов (обладает свойством полилинейности) с коэффициентами, зависящими от двух параметров ребер и инвариантными относительно дискретно действующей группы $D_{4}$ симметрий квадрата:

$$
\begin{aligned}
A\left(x, x_{1}, x_{2}, x_{12} ; \alpha_{1}, \alpha_{2}\right) & =\varepsilon A\left(x, x_{2}, x_{1}, x_{12} ; \alpha_{2}, \alpha_{1}\right)= \\
& =\sigma A\left(x_{1}, x, x_{12}, x_{2} ; \alpha_{1}, \alpha_{2}\right), \quad \varepsilon, \sigma= \pm 1 .
\end{aligned}
$$

Эта упрощающая гипотеза представляет собой так называемое cвойство mempaэдра, из которого следует, что при произвольных начальных значениях $x, x_{1}, x_{2}$ и $x_{3}$ функция $x_{123} \doteq x_{123}\left(x, x_{1}, x_{2}, x_{3} ; \alpha_{1}, \alpha_{2}, \alpha_{3}\right)$ не зависит от $x$. На трехмерной 


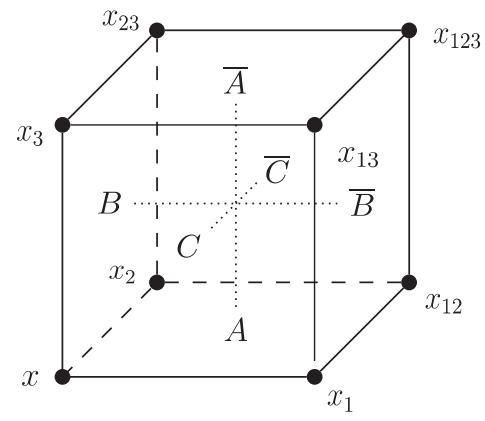

Рис. 2. Уравнения куба.

решетке индексов $m, n$ и $p$, на кубе, изображенном на рис. 2 , приняты следующие обозначения: $x=x_{m, n, p}, x_{1}=x_{m+1, n, p}, x_{2}=x_{m, n+1, p}$ и $x_{3}=x_{m, n, p+1}$. Тогда $x_{123}=x_{m+1, n+1, p+1}$. Уравнение решетки

$$
A\left(x_{m, n, p}, x_{m+1, n, p}, x_{m, n+1, p}, x_{m+1, n+1, p} ; \alpha_{1}(m), \alpha_{2}(n)\right)=0
$$

выполняется для каждого квадрата решетки индексов $m$ и $n$ и зависит параметрически от индекса $p$, а уравнение $\bar{A}=0$ имеет ту же структуру относительно индексов $m$ и $n$, что и уравнение $A=0$, но при других значениях $p, p+1$. Уравнение $B=0$ определено на квадратах решетки индексов $m$ и $p$ и зависит параметрически от индекса $n$, а уравнение $\bar{B}=0$ определено на квадратах решетки индексов $n$ и $p$ и параметрически зависит от индекса $m$. Результат классификации состоит в составлении набора двух списков уравнений $(H$ и $Q)$ для в общей сложности семи согласованных систем уравнений (с точностью до общего преобразования Мёбиуса переменных полей и точечных преобразований параметров ребер), представленных в работе [9]. Таким образом, на рис. 2, изображающем двумерную решетку, $\bar{A}$ задает копию уравнения $A$ (т. е. одно из семи уравнений $H$ и $Q$ ), а на гранях $B$ и $\bar{B}$ устанавливаются соотношения между решением уравнения $A$ и решениями уравнения $\bar{A}$, т. е. осуществляется автопреобразование Беклунда для уравнения $A$. При переходе в проективное пространство из автопреобразования Беклунда получается пара Лакса.

Теми же самыми авторами в работе [10] рассматривалась более общая перспектива развития задачи классификации. Там предполагалось, что на гранях $A, B, C$ и $\bar{A}$, $\bar{B}$ и $\bar{C}$ куба согласованности могут задаваться априори различные квад-уравнения без предположения о наличии какой-либо $D_{4}$-симметрии или свойства тетраэдра. Авторы указанной работы рассматривали наборы из шести (априори различных) квад-уравнений, ассоциированных с гранями трехмерного куба (см. рис. 2):

$$
\begin{array}{ll}
A\left(x, x_{1}, x_{2}, x_{12} ; \alpha_{1}, \alpha_{2}\right)=0, & \bar{A}\left(x_{3}, x_{13}, x_{23}, x_{123} ; \alpha_{1}, \alpha_{2}\right)=0, \\
B\left(x, x_{2}, x_{3}, x_{23}, \alpha_{2}, \alpha_{3}\right)=0, & \bar{B}\left(x_{1}, x_{12}, x_{13}, x_{123}, \alpha_{2}, \alpha_{3}\right)=0, \\
C\left(x, x_{1}, x_{3}, x_{13} ; \alpha_{1}, \alpha_{3}\right)=0, & \bar{C}\left(x_{2}, x_{12}, x_{23}, x_{123} ; \alpha_{1}, \alpha_{3}\right)=0 .
\end{array}
$$

Такой набор из шести уравнений оказывается трехмерно согласованным, если при произвольных начальных данных $x, x_{1}, x_{2}$ и $x_{3}$ три значения величины $x_{123}$, вычисленные с помощью уравнений $\bar{A}=0, \bar{B}=0$ и $\bar{C}=0$, совпадают. В результате 
авторы работы [10] воспроизвели уравнения $Q$-типа из работы [9] и привели некоторые новые примеры квад-уравнений типа $H$, которые оказались деформациями соответствующего уравнения, полученного в работе [9].

Болл [11]-[13] классифицировал все согласованные квад-уравнения, обладающие свойством тетраэдра, в отсутствие каких-либо дополнительных предположений. Его результаты были сформулированы в работе [13] в виде набора теорем, начиная с теоремы 3.9 и заканчивая теоремой 3.14 , в которых перечислены все согласованные шестичленные конфигурации с точностью до преобразований из $(\mathrm{Möb})^{8}-$ группы независимых преобразований Мёбиуса восьми полей в вершинах куба согласованности. Задавая для каждого из уравнений (1) соответствующие биквадраты

$$
A^{i, j} \equiv A^{i, j}\left(x_{i}, x_{j}\right)=A_{x_{m}} A_{, x_{n}}-A A_{x_{m} x_{n}}
$$

где $\{m, n\}$ - дополнение к набору индексов $\{i, j\}$ в множестве $\{1,2,3,4\}$, можно разбить все квад-уравнения на три несвязных семейства: уравнения $Q$-типа (без вырожденных биквадратов), уравнения $H^{4}$-типа (с четырьмя вырожденными биквадратами) и уравнения $H^{6}$-типа (все шесть биквадратов вырождены).

Следует отметить, что все классификационные результаты выполняются локально в том смысле, что все они относятся к отдельной квадратной ячейке или к отдельному кубу. Важная задача вложения этих результатов в двух- или трехмерную решетку отдельных уравнений квадратов или кубов с сохранением условия трехмерной согласованности уже обсуждалась в работе [10] с помощью концепции черно-белой $(\mathrm{BW})$ решетки. Одним из путей решения этой задачи оказывается идея вложить уравнения (2) в $\mathbb{Z}^{2}$-решетку, элементарная ячейка которой имеет размер выше единицы. В таком случае уравнение $Q=Q\left(x, x_{1}, x_{2}, x_{12} ; \alpha_{1}, \alpha_{2}\right)$ продолжается на решетку, а уравнения решетки становятся интегрируемыми или линеаризуемыми. Для этого, следуя работе [11], отобразим квадрат симметрично относительно его правой и верхней сторон, а затем завершим построение двумерной решетки, снова отобразив одно из полученных таким образом уравнений симметрично относительно другой из выбранных выше сторон ${ }^{1)}$. Эта процедура представлена в графическом виде на рис. 3 , а на уровне квад-уравнений это отвечает построению трех уравнений, получаемых из уравнения $Q=Q\left(x, x_{1}, x_{2}, x_{12} ; \alpha_{1}, \alpha_{2}\right)$ переворотом одного из участвующих в нем полей:

$$
\begin{aligned}
Q & =Q\left(x, x_{1}, x_{2}, x_{12}, \alpha_{1}, \alpha_{2}\right)=0, \\
Q Q & =Q\left(x_{1}, x, x_{12}, x_{2}, \alpha_{1}, \alpha_{2}\right)=0, \\
\underline{Q} & =Q\left(x_{2}, x_{12}, x, x_{1}, \alpha_{1}, \alpha_{2}\right)=0, \\
\underline{\underline{Q}} & =Q\left(x_{12}, x_{2}, x_{1}, x, \alpha_{1}, \alpha_{2}\right)=0 .
\end{aligned}
$$

Замащивая полную решетку $\mathbb{Z}^{2}$ такими уравнениями, получим уравнение в частных конечных разностях, которое можно исследовать с помощью известных методов. Поскольку априори $Q \neq|Q \neq \underline{Q} \neq| \underline{Q}$, построенная таким образом решетка будет раскрашена в четыре цвета, т. е. получается обобщение BW-решетки [14], [15].

1) Отметим, что вне зависимости от выбора стороны квадрата результат последнего отражения оказывается одним и тем же. 


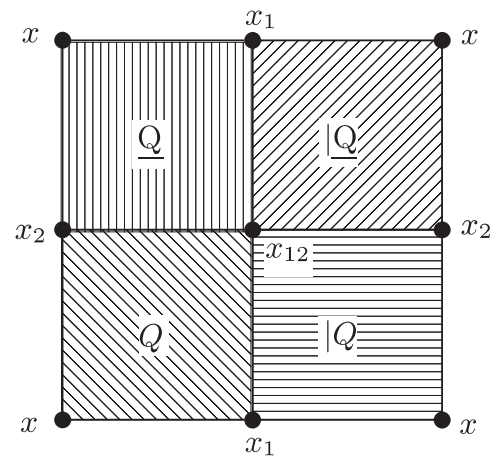

Рис. 3. Четырехцветная решетка.

В случае, когда уравнение $Q=0$ инвариантно относительно действия группы $D_{4}$, получаем

$$
Q=|Q=\underline{Q}=| \underline{Q},
$$

откуда вытекает, что элементарная ячейка имеет единичный размер и имеет место классификация Адлера-Бобенко-Суриса. Когда

$$
\begin{aligned}
Q\left(x, x_{1}, x_{2}, x_{12}, \alpha_{1}, \alpha_{2}\right) & =\sigma Q\left(x, x_{2}, x_{1}, x_{12}, \alpha_{2}, \alpha_{1}\right)= \\
& =\varepsilon Q\left(x_{12}, x_{1}, x_{2}, x, \alpha_{2}, \alpha_{1}\right), \quad \sigma, \varepsilon \in\{ \pm 1\},
\end{aligned}
$$

T. e.

$$
Q=|Q, \quad \underline{Q}=| Q,
$$

уравнение обладает ромбической симметрией, а если

$$
Q\left(x, x_{1}, x_{2}, x_{12}, \alpha_{1}, \alpha_{2}\right)=Q\left(x_{1}, x, x_{12}, x_{2}, \alpha_{1}, \alpha_{2}\right),
$$

T. e.

$$
Q=|Q, \quad \underline{Q}=| \underline{Q}
$$

или

$$
Q=\underline{Q}, \quad|Q=| \underline{Q},
$$

будем говорить, что уравнение имеет трапецеидальную симметрию. Подробное исследование всех решеток, полученных из ромбического $H^{4}$-семейства, включая построение их пар Лакса, преобразований Беклунда и бесконечных иерархий обобщенных симметрий, было произведено в работе [15]. Авторы работы [16] исследовали все уравнения из классификации Болла, не рассмотренные ранее в литератуpe [9], [10], [15], показали их линеаризуемость с помощью теста интегрируемости на основе алгебраической энтропии [17], [18] и нашли их линеаризации в явном виде. Больше подробностей о линеаризуемости можно найти в работах [19], [20].

Общая процедура вложения представлена в работах [11], [13]. Различные вложения в трехмерные согласованные решетки, приводящие либо к интегрируемым, либо к неинтегрируемым уравнениям, изучались в работе [14] с помощью анализа на основе алгебраической энтропии. 
После оригинальной работы Адлера, Бобенко и Суриса [9] были предприняты различные попытки упростить требования, налагаемые на согласованные квад-уравнения. На этом пути в работах [21], [22] были получены четыре нететраэдральных модели, три из которых обладали $D_{4}$-симметрией. Все эти модели или задаются линейными уравнениями, или (более-менее тривиально) линеаризуются [23]. Другие нететраэдральные согласованные системы линейных уравнений на квадратичной решетке исследовались в работах [10], [24].

Существование ложных пар Лакса хорошо известно, но мы еще далеки от полного понимания этого явления. Термин пары Лакса относится к паре линейных уравнений (переопределенная система), связанных с нелинейной интегрируемой системой через условие совместности. Наиболее важным свойством пары Лакса оказывается то, что она должна доказывать интегрируемость связанной с ней нелинейной системы и содержать информацию о нетривиальных решениях такой системы.

Ложные пары Лакса - это такие пары Лакса, которые не доказывают интегрируемость связанных с ними нелинейных систем. Ложные пары Лакса часто имеют вид, весьма сходный с видом, характерным для пар Лакса, обеспечивающих интегрируемость, и некритически настроенные эксперты в области интегрируемых систем продолжают публиковать примеры ложных пар Лакса, которые они принимают за настоящие (см. примеры в работе [25]). Более того, ложные пары Лакса обычно связываются с линеаризуемыми системами, так что доказательство ложности пары Лакса оказывается тем не менее способом доказать линеаризуемость системы.

Пары Лакса могут быть дискретными или непрерывными, матричными или скалярными, применяемыми в методе обратной задачи рассеяния или в изомонодромных деформациях, и ложные пары Лакса возникают во всех этих категориях. Хотя в литературе имеется множество ссылок на ложные пары Лакса, одной из наиболее известных является работа [26]; гораздо менее обширная литература посвящена объяснению явления ложных пар Лакса и тому, как их распознавать. Тем не менее для распознавания ложных пар Лакса придуманы разнообразные методы (см. [25], [27]-[33]).

Ниже в разделе 2 рассматриваются простейшие трапецеидальные $H^{4}$-уравнения, а именно ${ }_{t} H_{1}^{\varepsilon}$-уравнение, для которого строится пара Лакса. В разделе 3 приведено построение непосредственной линеаризации этого уравнения и показано, что полученная таким образом пара Лакса на самом деле оказывается эффективно ложной. В разделе 4 рассматриваются обобщенные симметрии этого уравнения, которые оказываются дифференциально-разностными уравнениями, допускающими по построению преобразования Беклунда. Выводы содержатся в разделе 5.

\section{2. ${ }_{t} H_{1}^{\varepsilon}$-УРАВНЕНИЕ}

Прежде чем определить уравнение ${ }_{t} H_{1}^{\varepsilon}$, необходимо ввести обозначения:

$$
\mathcal{F}_{p}^{( \pm)} \doteq \frac{1 \pm(-1)^{p}}{2}, \quad \alpha_{1} \doteq \alpha_{1}(m), \quad \alpha_{2} \doteq \alpha_{2}(n), \quad \alpha_{3} \doteq \alpha_{3}(p),
$$

где $\alpha_{1}, \alpha_{2}$ - постоянные, которые могут нести зависимость от переменной решетки и играют роль шага решетки при переходе к непрерывному пределу. Постоянная $\alpha_{3}$ 
возникает в преобразованиях Беклунда и играет роль спектрального параметра:

$$
\begin{gathered}
\psi_{m, n+1}=\ell_{m, n} \cdot L_{m, n} \psi_{m, n}, \quad \psi_{m+1, n}=\mathrm{m}_{m, n} \cdot M_{m, n} \psi_{m, n}, \\
\tau_{m, n} \cdot L_{m+1, n} \cdot M_{m, n}-M_{m, n+1} \cdot L_{m, n}=0, \quad \tau_{m, n} \doteq \frac{\mathrm{m}_{m, n} \ell_{m+1, n}}{\ell_{m, n} \mathrm{~m}_{m, n+1}}, \\
\ell_{m, n} \doteq \ell\left(m, n, x_{m, n}, x_{m, n+1} ; \alpha_{3}, \alpha_{1}, \varepsilon\right), \quad L_{m, n} \doteq L\left(m, n, x_{m, n}, x_{m, n+1} ; \alpha_{3}, \alpha_{1}, \varepsilon\right), \\
\mathrm{m}_{m, n} \doteq \mathrm{m}\left(m, n, x_{m, n}, x_{m+1, n} ; \alpha_{2}, \alpha_{3}, \varepsilon\right), \quad M_{m, n} \doteq M\left(m, n, x_{m, n}, x_{m+1, n} ; \alpha_{2}, \alpha_{3}, \varepsilon\right) .
\end{gathered}
$$

Элементы $L$ и $M$ задают пару Лакса на решетке индексов $m$ и $n$, а уравнения (11) представляют соответствующее уравнение Лакса на решетке. Связанные с ним коэффициенты нормировки обозначены через $\ell$ и $\mathrm{m}$, а $\tau$ есть их отношение, появляющееся в уравнении Лакса.

Теперь запишем ${ }_{t} H_{1}^{\varepsilon}$-уравнение:

$$
\begin{aligned}
& \left(x_{m, n}-x_{m+1, n}\right)\left(x_{m, n+1}-x_{m+1, n+1}\right)-\alpha_{2}- \\
& \quad-\varepsilon^{2} \alpha_{2}\left(\mathcal{F}_{n}^{(+)} x_{m, n+1} x_{m+1, n+1}+\mathcal{F}_{n}^{(-)} x_{m, n} x_{m+1, n}\right)=0,
\end{aligned}
$$

где $\varepsilon$ - произвольная константа. Уравнение (12) получается из уравнения на ячейке

$$
\left(x-x_{2}\right)\left(x_{3}-x_{23}\right)-\alpha_{2}\left(1+\varepsilon^{2} x_{3} x_{23}\right)=0
$$

с помощью процедуры, схематически описанной в разделе 1 и представленной в деталях в работе [16].

Пара Лакса этого уравнения и его коэффициенты нормировки имеют вид

$$
\begin{aligned}
L_{m, n} & =\left(\begin{array}{cc}
x_{m, n+1} & -x_{m, n} x_{m, n+1}+\alpha_{3} \\
1 & -x_{m, n}
\end{array}\right)-\varepsilon^{2} \alpha_{3}\left(\begin{array}{cc}
-\mathcal{F}_{n}^{(-)} x_{m, n} & 0 \\
0 & \mathcal{F}_{n}^{(+)} x_{m, n+1}
\end{array}\right), \\
M_{m, n} & =\left(\begin{array}{cc}
\alpha_{3}\left(x_{m, n}-x_{m+1, n}\right)+\alpha_{2} x_{m+1, n} & -\alpha_{2} x_{m, n} x_{m+1, n}-\varepsilon^{2} \alpha_{3} \alpha_{2}\left(\alpha_{3}-\alpha_{2}\right) \mathcal{F}_{n}^{(+)} \\
\alpha_{2} & \alpha_{1}\left(x_{m, n}-x_{m+1, n}\right)-\alpha_{2} x_{m, n}
\end{array}\right), \\
\tau_{m, n} & =\frac{\alpha_{2}\left(1+\varepsilon^{2} \mathcal{F}_{n}^{(-)} x_{m, n}^{2}+\varepsilon^{2} \mathcal{F}_{n}^{(+)} x_{m, n+1}^{2}\right)}{\left(x_{m, n}-x_{m+1, n}\right)^{2}+\varepsilon^{2} \alpha_{2}^{2} \mathcal{F}_{n}^{(+)}}, \\
\ell_{m, n} & =\frac{1}{1-i \varepsilon\left(\mathcal{F}_{n}^{(-)} x_{m, n}-\mathcal{F}_{n}^{(+)} x_{m, n+1}\right)}, \\
\mathrm{m} & =\frac{(14 \mathrm{~b})}{x_{m, n}-x_{m+1, n}+i \varepsilon \alpha_{2} \mathcal{F}_{n}^{(+)}} .
\end{aligned}
$$

Здесь с помощью весьма нетривиального вычисления удается переписать формулы для связности (14c), (14d), (14е) без применения стандартных членов с квадратными корнями. Уравнения, связанные с другими сторонами куба, имеют вид

$$
\begin{aligned}
& \alpha_{2}\left(x_{m, p}-x_{m, p+1}\right)\left(x_{m+1, p}-x_{m+1, p+1}\right)+\varepsilon^{2} \alpha_{3} \alpha_{2}\left(\alpha_{3}-\alpha_{2}\right) \mathcal{F}_{q}^{(+)}- \\
& \quad-\alpha_{3}\left(x_{m, p}-x_{m+1, p}\right)\left(x_{m, p+1}-x_{m+1, p+1}\right)=0 \\
& \left(x_{n, q}-x_{n, q+1}\right)\left(x_{n+1, q}-x_{n+1, q+1}\right)-\alpha_{3}- \\
& \quad-\varepsilon^{2} \alpha_{3}\left(\mathcal{F}_{n}^{(+)} x_{n+1, q} x_{n+1, q+1}+\mathcal{F}_{n}^{(-)} x_{n, q} x_{n, q+1}\right)=0 .
\end{aligned}
$$


Уравнения (15) суть преобразования Беклунда для уравнения ${ }_{t} H_{1}^{\varepsilon}$, в которых переменные решетки $p+1$ и $q+1$ интерпретируются как индексы, параметризующие новое решение. При переходе в проективное пространство уравнения (15) приводят к паре Лакса (14a), (14b).

\section{3. ЛИНЕАРИЗАЦИЯ И ЛОЖНЫЕ ПАРЫ ЛАКСА}

Перейдем к анализу уравнения (12). В нем всегда можно положить $\alpha_{2} \neq 0$, иначе это уравнение вырождается в уравнение

$$
\left(x_{m, n}-x_{m+1, n}\right)\left(x_{m, n+1}-x_{m+1, n+1}\right)=0,
$$

решение которого на решетке оказывается тривиальным. Введем обозначения $x_{m, 2 k} \doteq w_{m, k}, x_{m, 2 k+1} \doteq z_{m, k}$. Тогда уравнение (12) приводится к следующей системе двух связанных автономных разностных уравнений:

$$
\begin{gathered}
\mathcal{E}_{m} \equiv\left(w_{m, k}-w_{m+1, k}\right)\left(z_{m, k}-z_{m+1, k}\right)-\varepsilon^{2} \alpha_{2} z_{m, k} z_{m+1, k}-\alpha_{2}=0, \\
\left(w_{m, k+1}-w_{m+1, k+1}\right)\left(z_{m, k}-z_{m+1, k}\right)-\varepsilon^{2} \alpha_{2} z_{m, k} z_{m+1, k}-\alpha_{2}=0 .
\end{gathered}
$$

Вычитая уравнение (16b) из (16a), получим

$$
\left(w_{m, k}-w_{m+1, k}-w_{m, k+1}+w_{m+1, k+1}\right)\left(z_{m, k}-z_{m+1, k}\right)=0 .
$$

Теперь возможны два случая.

Случай 1. Если $z_{m, k}=f_{k}$, где $f_{k}$ - функция общего вида, то уравнение (17) выполнено. Из (16a) или из (16b) имеем $\varepsilon \neq 0$, так что, решая уравнение относительно $f_{k}$, получим

$$
f_{k}= \pm \frac{i}{\varepsilon}
$$

Случай 2. Если $z_{m, k} \neq f_{k}$, где $f_{k}$ определено в (18), тогда $w_{m, k}=g_{m}+h_{k}$, где $g_{m}$ и $h_{k}$ - произвольные функции своих аргументов. Следовательно, уравнения (16) сводятся к уравнению

$$
\varepsilon^{2} z_{m, k} z_{m+1, k}+\kappa_{m}\left(z_{m, k}-z_{m+1, k}\right)+1=0, \quad \kappa_{m} \doteq \frac{g_{m+1}-g_{m}}{\alpha_{2}} .
$$

На этом этапе возникают два подслучая.

Подслучай 2.1. Если $\varepsilon=0$, то из (19) следует, что $\kappa_{m} \neq 0$. Решая это уравнение, получим

$$
z_{m+1, k}-z_{m, k}=\frac{1}{\kappa_{m}}
$$

и, следовательно,

$$
\begin{array}{rlrl}
z_{m, k} & =j_{k}+\sum_{l=m_{0}}^{m-1} \frac{1}{\kappa_{l}}, \quad & & m \geqslant m_{0}+1, \\
z_{m, k} & =j_{k}-\sum_{l=m}^{m_{0}-1} \frac{1}{\kappa_{l}}, &
\end{array}
$$


где $j_{k}=z_{m_{0}, k}-$ произвольная константа интегрирования, представляющая собой функцию своих аргументов.

Подслучай 2.2. Если $\varepsilon \neq 0$, уравнение (19) принимает вид дискретного уравнения Риккати, линеаризуемого преобразованием Мёбиуса $z_{m, k} \doteq(i / \varepsilon)\left(\left(y_{m, k}-1\right) /\left(y_{m, k}+1\right)\right)$, приводящим к уравнению

$$
\left(i \kappa_{m}-\varepsilon\right) y_{m+1, k}=\left(i \kappa_{m}+\varepsilon\right) y_{m, k} .
$$

Так как $\kappa_{m} \neq \pm i \varepsilon$, поскольку иначе $y_{m, k}=0$ и $z_{m, k}=-i / \varepsilon$, из условия (22) получаем

$$
\begin{array}{rlr}
y_{m, k} & =j_{k} \prod_{l=m_{0}}^{m-1} \frac{i \kappa_{l}+\varepsilon}{i \kappa_{l}-\varepsilon}, \quad m \geqslant m_{0}+1, \\
y_{m, k} & =j_{k} \prod_{l=m}^{m_{0}-1} \frac{i \kappa_{l}-\varepsilon}{i \kappa_{l}+\varepsilon}, \quad m \leqslant m_{0}-1,
\end{array}
$$

где $j_{k}=y_{m_{0}, k}$ - другая константа интегрирования, являющаяся функцией своих аргументов.

Тем самым всегда можно получить полное решение исходной системы.

Заметим, что при $\varepsilon=0$ уравнение (12) принимает вид

$$
\left(x_{m, n}-x_{m+1, n}\right)\left(x_{m, n+1}-x_{m+1, n+1}\right)-\alpha_{2}=0,
$$

и контактное преобразование типа преобразования Мёбиуса

$$
x_{m, n}-x_{m+1, n}=\sqrt{\alpha_{2}} \frac{2 w_{m, n}+1-\alpha_{2}}{2 w_{m, n}+1+\alpha_{2}}
$$

переводит уравнение (12) в следующее линейное уравнение первого порядка:

$$
w_{m, n+1}+w_{m, n}+1=0,
$$

решение которого имеет вид

$$
x_{m, n}=x_{0, n}-\sqrt{\alpha_{2}} \sum_{l=1}^{m} \frac{2 w_{l, 0}(-1)^{n}+(-1)^{n}-\alpha_{2}}{2 w_{l, 0}(-1)^{n}+(-1)^{n}+\alpha_{2}} .
$$

Здесь $x_{0, n}$ и $w_{m, 0}$ суть две произвольные константы интегрирования, задаваемые функциями своих аргументов. Другое линеаризующее преобразование при $\varepsilon=0$ задается формулой

$$
x_{m, n}-x_{m+1, n}=\sqrt{\alpha_{2}} e^{z_{m, n}},
$$

оно переводит уравнение (12) в следующее линейное уравнение первого порядка:

$$
z_{m, n+1}+z_{m, n}=2 \pi \kappa, \quad k=0,1 .
$$

Линеаризацию можно произвести также на основе пары Лакса (14). Вводя поля $\rho_{m, k} \doteq \tilde{\psi}_{m, 2 k}^{(1)}, \sigma_{m, k} \doteq \tilde{\psi}_{m, 2 k+1}^{(1)}, \theta_{m, k} \doteq \tilde{\psi}_{m, 2 k}^{(2)}, \chi_{m, k} \doteq \tilde{\psi}_{m, 2 k+1}^{(2)}$, можно переписать пару 
Лакса в виде

$$
\begin{gathered}
\phi_{m, k+1}=\mathcal{L}_{m, k} \phi_{m, k}, \quad \phi_{m+1, k}=\mathcal{M}_{m, k} \phi_{m, k}, \quad \phi_{m, k} \doteq\left(\begin{array}{c}
\rho_{m, k} \\
\theta_{m, k}
\end{array}\right), \\
\mathcal{L}_{m, k}=-\alpha_{3}\left(\begin{array}{cc}
1 & w_{m, k+1}-w_{m, k} \\
0 & 1
\end{array}\right), \\
\mathcal{M}_{m, k}=\frac{1}{w_{m, k}-w_{m+1, k}+i \varepsilon \alpha_{2}} \times \\
\times\left(\begin{array}{rrr}
\alpha_{3}\left(w_{m, k}-w_{m+1, k}\right)+\alpha_{2} w_{m+1, k} & -\alpha_{2} w_{m, k} w_{m+1, k}-\varepsilon^{2} \alpha_{3} \alpha_{2}\left(\alpha_{3}-\alpha_{2}\right) \\
\alpha_{2} & \alpha_{1}\left(w_{m, k}-w_{m+1, k}\right)-\alpha_{2} w_{m, k}
\end{array}\right), \\
\left(\begin{array}{c}
\sigma_{m, k} \\
\chi_{m, k}
\end{array}\right)=\frac{1}{i-\varepsilon z_{m, k}}\left(\begin{array}{rr}
z_{m, k} & \alpha_{3}-w_{m, k} z_{q, k} \\
1 & -w_{m, k}-\varepsilon^{2} \alpha_{3} z_{m, k}
\end{array}\right) \phi_{m, k}, \\
\alpha_{3} \mathcal{E}_{m} \mathcal{N}_{q, k} \phi_{q, k}=0, \quad \mathcal{N}_{m, k} \doteq\left(\begin{array}{rr}
i \varepsilon\left(z_{m, k}-z_{m+1, k}\right) & n_{12} \\
z_{m, k}-z_{m+1, k} & n_{22}
\end{array}\right),
\end{gathered}
$$

где

$$
\begin{aligned}
n_{12}= & i \varepsilon\left(w_{m, k} z_{m+1, k}-w_{m+1, k} z_{m, k}\right)+\varepsilon^{2} \alpha_{3}\left(z_{m, k}-z_{m+1, k}\right)- \\
& \quad-\varepsilon^{2} \alpha_{2} z_{m, k}+w_{m, k}-w_{m+1, k}+i \varepsilon \alpha_{2}, \\
n_{22}=w_{m+1, k} z_{m+1, k}-w_{m, k} z_{m, k}-i \varepsilon \alpha_{3}\left(z_{m, k}-z_{m+1, k}\right)-i \varepsilon \alpha_{2} z_{m+1, k}- & \quad-i \varepsilon\left(w_{m, k}-w_{m+1, k}+i \varepsilon \alpha_{2}\right) z_{m, k} z_{m+1, k}
\end{aligned}
$$

$w_{m, k}-w_{m+1, k}+i \varepsilon \alpha_{2} \neq 0, z_{m, k}-z_{m+1, k} \neq 0$, а $\mathcal{E}_{m}-$ левая часть уравнения (16а). При выводе $(30 \mathrm{a})$ и $(30 \mathrm{~d})$ применялось соотношение $(30 \mathrm{c})$ и разностные уравнения, из него вытекающие. Условие совместности уравнений (30a) и (30b) требует выполнения соотношения

$$
w_{m, k}-w_{m+1, k}-w_{m, k+1}+w_{m+1, k+1}=0,
$$

а из $(30 \mathrm{~d})$ следует, что $\mathcal{E}_{m}=0$. Аналогично можно записать все соотношения в терминах вектора $\left(\begin{array}{l}\sigma_{m, k} \\ \chi_{m, k}\end{array}\right)$. В этом случае совместность построенной таким образом пары Лакса после интегрирования приводит к условию (19).

Покажем теперь, что пара Лакса (30) является ложной. При калибровочном преобразовании

$$
\phi_{m, k}=\mathcal{G}_{m, k} \phi_{m, k}^{\prime}, \quad \mathcal{G}_{m, k} \doteq\left(-\alpha_{3}^{k}\right) \alpha_{1}^{m}\left(\begin{array}{cc}
1 & w_{m, k} \\
0 & 1
\end{array}\right),
$$

пара Лакса (30) принимает вид

$$
\begin{aligned}
\mathcal{L}_{m, k}^{\prime} & =\left(\begin{array}{ll}
1 & 0 \\
0 & 1
\end{array}\right) \\
\mathcal{M}_{m, k}^{\prime} & =\frac{1}{w_{m, k}-w_{m+1, k}+i \varepsilon \alpha_{2}} \times
\end{aligned}
$$




$$
\times\left(\begin{array}{cc}
w_{m, k}-w_{m+1, k} & \left(w_{m, k}-w_{m+1, k}\right)^{2}-\varepsilon^{2} \alpha_{2}\left(\alpha_{3}-\alpha_{2}\right) \\
\frac{\alpha_{2}}{\alpha_{3}} & w_{m, k}-w_{m+1, k}
\end{array}\right) .
$$

В формулах (31), (32) матрица $\mathcal{L}_{m, k}^{\prime}$ не зависит ни от спектрального параметра $\alpha_{3}$, ни от какого-либо из полей. Поэтому эта матрица не представляет интереса ни для какой спектральной задачи.

\section{4. ОБОБЩЕННЫЕ СИММЕТРИИ И ИХ ПРЕОБРАЗОВАНИЯ БЕКЛУНДА}

В этом разделе производится построение трехточечных обобщенных симметрий, представленных преобразованиями

$$
\begin{gathered}
x_{m, n ; \lambda}=g_{m, n}\left(s, t, u, v, \bar{u}, \bar{v}, x_{m, n} ; \alpha_{2}, \varepsilon\right), \quad s \doteq \frac{x_{m+1, n}-x_{m, n}}{1+\varepsilon^{2} x_{m+1, n} x_{m, n}} \\
t \doteq \frac{x_{m, n}-x_{m-1, n}}{1+\varepsilon^{2} x_{m-1, n} x_{m, n}}, \quad u \doteq x_{m+1, n}-x_{m, n}, \quad v \doteq x_{m, n}-x_{m-1, n}, \\
\bar{u} \doteq x_{m, n+1}-x_{m, n}, \quad \bar{v} \doteq x_{m, n}-x_{m, n-1},
\end{gathered}
$$

связанных с уравнением (12) в направлении $(m, n)$, а затем уравнение (12) интерпретируется как преобразование Беклунда для построенного дифференциально-разностного уравнения. Здесь $\lambda$ играет роль группового параметра.

\section{1. Трехточечные обобщенные симметрии вдоль направления $m$.}

Случай $\varepsilon \neq 0$. В этом случае симметрии имеют вид

$$
\begin{aligned}
g_{m, n}^{(\varepsilon)}=\mathcal{F}_{n}^{(+)} & \left\{\frac{\alpha_{2}\left(v^{2}+\varepsilon^{2} \alpha_{2}^{2}\right)}{(u-v)(u+v)} B_{m}\left(\frac{\alpha_{2}}{u}\right)-\right. \\
& \left.-\frac{\alpha_{2}\left(u^{2}+\varepsilon^{2} \alpha_{2}^{2}\right)}{(u-v)(u+v)} B_{m-1}\left(\frac{\alpha_{2}}{v}\right)\left[x_{m, n}-\frac{\left(u^{2}+\varepsilon^{2} \alpha_{2}^{2}\right) v}{(u-v)(u+v)}\right] \omega+\gamma_{n}\right\}+ \\
+ & {\left[\frac{s^{2} t^{2}}{(s-t)(s+t)}\left(B_{m}(s)-B_{m-1}(t)\right)-\frac{s^{2} t}{(s-t)(s+t)} \omega+\delta_{n}\right] \times } \\
& \times \mathcal{F}_{n}^{(-)}\left(1+\varepsilon^{2} x_{m, n}^{2}\right),
\end{aligned}
$$

где $B_{m}(y), \gamma_{n}$ и $\delta_{n}-$ функции общего вида от своих аргументов, а $\omega$ - произвольная константа. Заметим, что любая из этих функций и константа могут в конечном итоге содержать зависимость от $\alpha_{2}$ и $\varepsilon$. Можно показать, что в случае $\varepsilon \neq 0$ не существует не зависящих от $n$ редукций приведенной выше симметрии, для которых $x_{m, n} \doteq x_{m}$.

Случай $\varepsilon=0$. В этом случае симметрии имеют более простой вид:

$$
\begin{aligned}
g_{m, n}^{(0)}= & \frac{u^{2} v^{2}}{(u-v)(u+v)}\left[\mathcal{F}_{n}^{(+)}\left(\frac{B_{m}\left(\alpha_{2} / u\right)}{u^{2}}-\frac{B_{m-1}\left(\alpha_{2} / v\right)}{v^{2}}\right) \alpha_{2}+\right. \\
& \left.+\mathcal{F}_{n}^{(-)}\left(B_{m}(u)-B_{m-1}(v)\right)\right]+\left[\frac{x_{m, n}}{2}-\frac{u^{2} v}{(u-v)(u+v)}\right] \omega+ \\
& +(-1)^{n} \sigma x_{m, n}+f_{n},
\end{aligned}
$$


где $B_{m}(y)$ и $f_{n}$ - функции общего вида от своих аргументов, а $\omega$ и $\sigma$ - произвольные константы. Как и в предыдущем случае, каждая из этих функций и констант может содержать зависимость от $\alpha_{2}$. Легко показать, что при выборе

$$
f_{n}=\frac{1+(-1)^{n}}{2} \gamma_{n}+\frac{1-(-1)^{n}}{2} \delta_{n}
$$

предел $\varepsilon \rightarrow 0$ симметрии (33) совпадает с ограничением $\sigma=\omega / 2$ симметрии (34), и, таким образом, симметрия при $\varepsilon=0$ становится не зависящей от $n$. Поэтому естественно спросить, содержит ли семейство симметрий (34) редукции, не зависящие от $n$. Такая редукция существует и задается формулами

$$
\begin{gathered}
g_{m, n}^{(0)}=\frac{u^{2} v^{2}}{(u-v)(u+v)}\left(\frac{B_{m}\left(u ; \alpha_{2}\right)}{u}-\frac{B_{m-1}\left(v ; \alpha_{2}\right)}{v}\right)+ \\
+\left[\frac{x_{m, n}}{2}-\frac{u^{2} v}{(u-v)(u+v)}\right] \omega+\chi
\end{gathered}
$$

где $\omega$ и $\chi$ - произвольные константы (зависящие в принципе от $\left.\alpha_{2}\right)$, а $B_{m}\left(y ; \alpha_{2}\right)-$ функция от своих аргументов, удовлетворяющая следующему функциональному уравнению:

$$
B_{m}\left(\frac{\alpha_{2}}{y} ; \alpha_{2}\right)=B_{m}\left(y ; \alpha_{2}\right) .
$$

Общее решение уравнения (36) имеет вид

$$
B_{m}\left(y ; \alpha_{2}\right)= \begin{cases}G_{m}\left(y ; \alpha_{2}\right), & y \in \mathcal{B}\left(\alpha_{2}\right) \subset \Omega\left(\alpha_{2}\right) \\ G_{m}\left(\frac{\alpha_{2}}{y} ; \alpha_{2}\right), & \frac{\alpha_{2}}{y} \in \mathcal{B}\left(\alpha_{2}\right)\end{cases}
$$

где $G_{m}\left(y ; \alpha_{2}\right)$ - функция общего вида от своих аргументов, через $\Omega\left(\alpha_{2}\right)$ обозначено подмножество комплексной плоскости

$$
\Omega\left(\alpha_{2}\right) \doteq\left\{z \in \mathbb{C}:|z|>\sqrt{\left|\alpha_{2}\right|} \cup z=\sqrt{\left|\alpha_{2}\right|} e^{i \theta_{z}}, \frac{\theta_{\alpha_{2}}}{2} \leqslant \theta_{z} \leqslant \frac{\theta_{\alpha_{2}}}{2}+\pi\right\},
$$

а $\mathcal{B}\left(\alpha_{2}\right)$ - произвольное подмножество $\Omega\left(\alpha_{2}\right)$. В частности, если $B_{m}\left(y ; \alpha_{2}\right)$ - аналитическая функция в кольце с центром в начале координат и с радиусами $r_{1}\left(\alpha_{2}\right)$ и $r_{2}\left(\alpha_{2}\right)$ с условием $r_{1}\left(\alpha_{2}\right)<\sqrt{\left|\alpha_{2}\right|}<r_{2}\left(\alpha_{2}\right)$, то нетрудно показать, что

$$
B_{m}\left(y ; \alpha_{2}\right)=U_{m}\left(\frac{\alpha_{2}}{y}+y ; \alpha_{2}\right),
$$

причем $U_{m}\left(y ; \alpha_{2}\right)$ оказывается аналитической функцией $y$ в некоторой области комплексной плоскости, но в остальном представляет собой функцию общего вида по всем своим аргументам. Например, если в (35) положить $\omega=0$ и выбрать $U_{m}\left(y ; \alpha_{2}\right)=-1$, то получим симметрию

$$
x_{m, n, \lambda}=\frac{\left(x_{m+1, n}-x_{m, n}\right)\left(x_{m, n}-x_{m-1, n}\right)}{x_{m+1, n}-x_{m-1, n}}+\chi,
$$

которая преобразованием сдвига $x_{m, n} \doteq u_{m, n}+\lambda \chi$ переводится в подкласс ямиловских дискретизаций уравнения Кричевера-Новикова [8]. 
4.2. Трехточечные обобщенные симметрии вдоль направления $n$. Имеются следующие симметрии в направлении $n$ :

$$
\begin{gathered}
g_{m, n}^{(\varepsilon)}=\mathcal{F}_{n}^{(+)}\left(B_{n}\left(\frac{\bar{u}+\bar{v}}{1+\varepsilon^{2} x_{m, n+1} x_{m, n-1}}\right)+\kappa_{n}\right)+ \\
+\mathcal{F}_{n}^{(-)}\left(1+\varepsilon^{2} x_{m, n}^{2}\right)\left(C_{n}(\bar{u}+\bar{v})+\lambda_{n}\right),
\end{gathered}
$$

где $B_{n}(y), C_{n}(y), \kappa_{n}$ и $\lambda_{n}-$ произвольные функции от своих аргументов. 
В случае $\varepsilon=0$ имеем

$$
g_{m, n}^{(0)}=D_{n}(\bar{u}+\bar{v})+(-1)^{n} \sigma x_{m, n}+\theta_{n},
$$

где $D_{n}(y)$ и $\theta_{n}$ - произвольные функции своих аргументов, а $\sigma$ - произвольный параметр. Можно непосредственно показать, что при выборе $D_{n}(y)=\mathcal{F}_{n}^{(+)} B_{n}(y)+$ $\mathcal{F}_{n}^{(-)} C_{n}(y)$ и $\theta_{n}=\mathcal{F}_{n}^{(+)} \kappa_{n}+\mathcal{F}_{n}^{(-)} \lambda_{n}$ предел при $\varepsilon \rightarrow 0$ симметрии (37) совпадает с ограничением $\sigma=0$ для симметрии (38).

\section{3. Дифференциально-разностное уравнение и преобразования Бек-} лунда. Можно интерпретировать (33) при $\varepsilon \neq 0$ как дифференциально-разностное уравнение относительно переменной $m$. Поскольку (33) зависит также от $n$, это возможно в случае зависимости только через $\mathcal{F}_{n}^{(+)}$и $\mathcal{F}_{n}^{(-)}$, так как только в этом случае можно избавиться от этой зависимости с помощью перехода к полям, зависящим только от четных или нечетных значений аргумента $n$. В этом случае следует положить $\gamma_{n}$ и $\delta_{n}$ равными нулю и определить новые поля $w_{m}$ и $z_{m}$ так же, как это было сделано в разделе 3. При этом уравнение (33) переходит в следующую систему уравнений:

$$
\begin{aligned}
w_{m ; \lambda}^{(\varepsilon)}= & \left\{\frac{\alpha_{2}\left(\tilde{v}^{2}+\varepsilon^{2} \alpha_{2}^{2}\right)}{(\tilde{u}-\tilde{v})(\tilde{u}+\tilde{v})} B_{m}\left(\frac{\alpha_{2}}{\tilde{u}}\right)-\frac{\alpha_{2}\left(\tilde{u}^{2}+\varepsilon^{2} \alpha_{2}^{2}\right)}{(\tilde{u}-\tilde{v})(\tilde{u}+\tilde{v})} B_{m-1}\left(\frac{\alpha_{2}}{\tilde{v}}\right)+\right. \\
& \left.+\left[w_{m}-\frac{\left(\tilde{u}^{2}+\varepsilon^{2} \alpha_{2}^{2}\right) \tilde{v}}{(\tilde{u}-\tilde{v})(\tilde{u}+\tilde{v})}\right] \alpha\right\}, \\
z_{m ; \lambda}^{(\varepsilon)}= & \left\{\frac{\tilde{s}^{2} \tilde{t}^{2}\left[B_{m}(\tilde{s})-B_{m-1}(\tilde{t})\right]}{(\tilde{s}-\tilde{t})(\tilde{s}+\tilde{t})}-\frac{\tilde{s}^{2} \tilde{t} \omega}{(\tilde{s}-\tilde{t})(\tilde{s}+\tilde{t})}\right\}\left(1+\varepsilon^{2} z_{m}^{2}\right),
\end{aligned}
$$

где

$$
\begin{array}{ll}
\tilde{s} \doteq \frac{z_{m+1}-z_{m}}{1+\varepsilon^{2} z_{m+1} z_{m}}, & \tilde{t} \doteq \frac{z_{m}-z_{m-1}}{1+\varepsilon^{2} z_{m-1} z_{m}}, \\
\tilde{u} \doteq w_{m+1}-w_{m}, & \tilde{v} \doteq w_{m}-w_{m-1} .
\end{array}
$$

Уравнения (16a), (16b) оказываются преобразованиями Беклунда системы (39), (40), связывающими решение $\left(w_{m}, z_{m}\right)$ с новым решением $\left(\widetilde{w}_{m}, \tilde{z}_{m}\right)$, т. е.

$$
\begin{aligned}
& \left(w_{m}-w_{m+1}\right)\left(z_{m}-z_{m+1}\right)-\alpha_{2} \varepsilon^{2} z_{m} z_{m+1}-\alpha_{2}=0, \\
& \left(\widetilde{w}_{m}-\widetilde{w}_{m+1}\right)\left(\tilde{z}_{m}-\tilde{z}_{m+1}\right)-\alpha_{2} \varepsilon^{2} \tilde{z}_{m} \tilde{z}_{m+1}-\alpha_{2}=0, \\
& \left(\widetilde{w}_{m}-\widetilde{w}_{m+1}\right)\left(z_{m}-z_{m+1}\right)-\alpha_{2} \varepsilon^{2} z_{m} z_{m+1}-\alpha_{2}=0 .
\end{aligned}
$$

Из системы (41) видно, что решения уравнений (39), (40) не являются независимыми, так как они связаны уравнением (41a). Поэтому, если задано решение $\left(w_{m}, z_{m}\right)$ двух уравнений (39), (40), решая уравнение (41c), можно найти $\widetilde{w}_{m}$, а из (41b) получим $\tilde{z}_{m}$.

Можно получить аналогичный результат в случае уравнения (34) при $\varepsilon=0$.

В случае симметрий переменной $n$ при $\kappa_{m}=\lambda_{m}=0$ зависимость от $m$ оказывается просто параметрической, так что рассмотренное выше явление не имеет места. При этом получаются единственное неавтономное дифференциально-разностное 
уравнение

$$
w_{n, \lambda}=\mathcal{F}_{n}^{(+)} B_{n}\left(\frac{w_{n+1}-w_{n-1}}{1+\varepsilon^{2} w_{n+1} w_{n-1}}\right)+\mathcal{F}_{n}^{(-)}\left(1+\varepsilon^{2} w_{n}^{2}\right) C_{n}\left(w_{n+1}-w_{n-1}\right)
$$

и единственное неавтономное преобразование Беклунда

$$
\left(w_{n}-\widetilde{w}_{n}\right)\left(w_{n+1}-\widetilde{w}_{n+1}\right)-\varepsilon^{2} \alpha_{2}\left(\mathcal{F}_{n}^{(+)} w_{n+1} \widetilde{w}_{n+1}+\mathcal{F}_{n}^{(-)} w_{n} \widetilde{w}_{n}\right)-\alpha_{2}=0 .
$$

\section{5. ЗАКЛЮЧЕНИЕ}

В работе представлены результаты, касающиеся линеаризации и симметрий неавтономных нелинейных уравнений в частных конечных разностях, принадлежащих классификации Болла квад-графических уравнений на решетке, уравнений типа ${ }_{t} H_{1}^{\varepsilon}$. В частности, предъявлена явная линеаризация этого уравнения, полученная при сведении уравнения ${ }_{t} H_{1}^{\varepsilon}$ к системе автономных уравнений в частных разностях, которые могут быть решены явно, и было показано, что пара Лакса этого уравнения оказывается ложной, т. е. с помощью калибровочного преобразования спектральная задача приводится к виду, не зависящему ни от спектрального параметра, ни от полей теории.

Можно построить трехточечные обобщенные симметрии, отражающие свойство линеаризуемости дискретного уравнения, поскольку эти симметрии задаются произвольными функциями непрерывной переменной и дискретного индекса решетки. Этот интересный частный результат будет исследован в последующих работах.

Также в будущем авторы надеются провести анализ остальных линеаризуемых уравнений из классификации Болла.

\section{Список литературы}

[1] D. Levi, R. Benguria, Proc. Nat. Acad. Sci. USA, 77:9 (1981), 5025-5027.

[2] J. J. C. Nimmo, W. K. Schief, Proc. Roy. Soc. London Ser. A, 453:1957 (1997), 255-279.

[3] A. Doliwa, P. M. Santini, Phys. Lett. A, 233:4-6 (1997), 365-372.

[4] A. I. Bobenko, Yu. B. Suris, Int. Math. Res. Not., 2002:11 (2002), 573-611.

[5] T. Bridgman, W. Hereman, G. R. W. Quispel, P. H. van der Kamp, Found. Comput. Math., 13:4 (2013), 517-544.

[6] F. W. Nijhoff, Phys. Lett. A, 297:1-2 (2002), 49-58.

[7] F. W. Nijhoff, A. Walker, Glasg. Math. J., 43A (2001), 109-123.

[8] R. Yamilov, J. Phys. A: Math. Gen., 39:45 (2006), R541-R623.

[9] V. E. Adler, A. I. Bobenko, Yu. B. Suris, Commun. Math. Phys., 233:3 (2003), 513-543.

[10] В. Э. Адлер, А. И. Бобенко, Ю. Б. Сурис, Функи. анализ и его прил., 43:1 (2009), 3-21.

[11] R. Boll, J. Nonlinear Math. Phys., 18:3 (2011), 337-365.

[12] R. Boll, J. Nonlinear Math. Phys., 19:4 (2012), 1292001, 3 pp.

[13] R. Boll, Classification and Lagrangian structure of $3 D$ consistent quad-equations, Ph. D. thesis, Technische Universität, Berlin, 2012.

[14] J. Hietarinta, C. Viallet, Nonlinearity, 25:7 (2012), 1955-1966.

[15] P. D. Xenitidis, V. G. Papageorgiou, J. Phys. A: Math. Theor., 42:45 (2009), 454025, 13 pp.

[16] G. Gubbiotti, C. Scimiterna, D. Levi, J. Nonlinear Math. Phys., 23:4 (2016), 507-543.

[17] S. Tremblay, B. Grammaticos, A. Ramani, Phys. Lett. A, 278:6 (2001), 319-324.

[18] C. Viallet, Algebraic entropy for lattice equations, arXiv: math-ph/0609043. 
[19] G. Gubbiotti, R. I. Yamilov, Darboux integrability of trapezoidal $H^{4}$ and $H^{6}$ families of lattice equations I: First integrals, arXiv: 1608.03506.

[20] G. Gubbiotti, C. Scimitema, R. I. Yamilov, Darboux integrability of trapezoidal $H^{4}$ and $H^{6}$ families of lattice equations II: First integrals (in preparation).

[21] J. Hietarinta, J. Phys. A: Math. Gen., 37:6 (2004), L67-L73.

[22] J. Hietarinta, J. Nonlinear Math. Phys., 12, supp. 2 (2005), 223-230.

[23] A. Ramani, N. Joshi, B. Grammaticos, T. Tamizhmani, J. Phys. A: Math. Gen., 39:8 (2006), L145-L149.

[24] J. Atkinson, J. Phys. A: Math. Theor., 42:45 (2009), 454005, 7 pp.

[25] M. Hay, S. Butler, Simple identification of fake Lax pair, arXiv: 1311.2406.

[26] F. Calogero, M. C. Nucci, J. Math. Phys., 32:1 (1991), 72-74.

[27] D. Levi, A. Sym, G. Z. Tu, A working algorithm to isolate integrable surfaces in $E^{3}$, preprint DF-INFN № 761, INFN, Roma, 1990.

[28] Y.Q. Li, B. Li, S. Y. Lou, Constraints for evolution equations with some special forms of Lax pairs and distinguishing Lax pairs by available constraints, arXiv: 1008.1375.

[29] M. Marvan, Acta Appl. Math., 72:1-2 (2002), 51-65.

[30] M. Marvan, Acta Appl. Math., 109:1 (2010), 239-255.

[31] M. Marvan, Acta Appl. Math., 83:1-2 (2004), 39-68.

[32] S. Yu. Sakovich, True and fake Lax pairs: how to distinguish them, arXiv: nlin.SI/0112027.

[33] S. Yu. Sakovich, Acta Appl. Math., 83:1 (2004), 69-83, arXiv: nlin/0212019. 